Bull. Mater. Sci., Vol. 22, No. 2, April 1999, pp. 129-132. (C) Indian Academy of Sciences.

\title{
Microscopic order parameter of two nematogenic compounds using X-rays
}

\author{
A P DIVYA, D REVANNASIDDAIAH, R SOMASHEKAR* and M S MADHAVA \\ Department of Studies in Physics, University of Mysore, Mysore 570 006, India
}

MS received 28 October 1998; revised 12 January 1999

\begin{abstract}
X-ray Laue type difraction patterns were recorded using the image plate system at different temperatures in the nematic phase for two nematogenic compounds. Micro-orientational order parameters have been computed using different methods. These are compared with the microscopic orientational order parameters obtained from refractive index data. Higher order parameters have also been computed and compared. Distribution function at various temperatures for the two nematogenic compounds from Deutsch method has been reported.
\end{abstract}

Keywords. Nematic; orientational order; liquid crystal.

\section{Introduction}

The orientational order parameter of any nematic liquid crystal determines its applicability in electro-optic displays and hence the continued interest amongst various investigators to compute the order parameter. This can be evaluated by various methods viz. optical, NMR, X-rays etc. The earlier works of the determination of the OP using X-rays are due to Falgueirettes (1959), Delord and Falgueirettes (1965), de Vries (1972), Leadbetter and Norris (1979), and Baumann et al (1989). The simplest approach is that of Leadbetter (1979) which leads to the classical formula:

$$
I(\phi)=\int_{0}^{\pi / 2} f(\beta) \sec ^{2}(\phi)\left(\tan ^{2} \beta-\tan ^{2} \phi\right)^{-1 / 2} \sin \beta \mathrm{d} \beta,
$$

which relates the scattered intensities at an angle $\phi$ to the orientational distribution function $f(\beta)$ of the molecular axes. To evaluate the orientational distribution function $f(\beta)$ and the order parameter, various numerical and series expansion methods (see for e.g. Leadbetter and Wrighton 1979; Haase et al 1988; Fan et al 1990; Kelkar and Paranjape 1987; Bhattacharjee et al 1981) had been employed. Deutsch (1991) has shown that (1) can be analytically inverted which leads to general expressions for the order and higher order parameters. Davidson et al (1995) (here afterwards called Levelut method) have developed simple analytical calculation of (1) which leads to a series expression. Also, they have shown that single intensity measurement can lead to the

*Author for correspondence evaluation of $f(\beta)$. We have computed the order parameter by these methods for two nematogenic compounds viz. octylbenzoic acid (OBA) and nonylbenzoic acid (NBA) using good quality $\mathrm{X}$-ray intensity data. These results are compared with the order parameter obtained from refractive index data which have been already reported (Divya et al 1997).

\section{Experimental}

\subsection{Samples}

OBA and NBA were obtained from M/s Merck Ltd, UK which were purified by recrystallization in benzene. These compounds exhibit nematic phase and the nematic-isotropic transition temperatures are 112 and $115^{\circ} \mathrm{C}$ for $\mathrm{OBA}$ and NBA, respectively. The transition temperatures were determined by using a polarizing microscope in conjunction with a specially constructed hot stage. The observed values are in good agreement with the reported values.

\subsection{Collection of X-ray intensity data}

The samples were taken in capillary tubes and oriented by an external magnetic field ( 6 kilogauss). The X-ray intensity data from the sample was collected using Laue method at different temperatures in the nematic phase, the temperature being controlled to an accuracy of $\pm 0.011^{\circ} \mathrm{C}$. Using the supplied $\mathrm{X}$-ray software, the $\mathrm{X}$-ray intensity data were sorted as a function of the arc angle. The wavelength of the X-rays used was $1.5418 \AA(\mathrm{Cu} \mathrm{K} \alpha)$. No monochromator was used in this experiment. An image plate with a plate diameter of $18 \mathrm{~cm}$ was used 
as the detector to collect the intensity data. The intensity versus arc angle data were obtained by integrating the diffused reflections over an annular ring centred on these reflections. These recordings for $\mathrm{OBA}$ and NBA are given in figure 1 for a temperature in the nematic phase.

\section{Analysis of X-ray data}

Deutsch (1991) has derived an exact analytical solution to (1) and has obtained the expressions for the two lowest order parameters $\left\langle P_{2}\right\rangle$ and $\left\langle P_{4}\right\rangle$ as

$$
\begin{aligned}
\left\langle P_{2}\right\rangle= & 1-(3 / 2 N) \int_{0}^{\pi / 2} I(\phi)\left[\sin ^{2} \phi\right. \\
& +\sin \phi \cos \phi \ln [(1+\sin \phi) / \cos \phi\}] \mathrm{d} \phi,
\end{aligned}
$$

and

$$
\begin{aligned}
\left\langle P_{4}\right\rangle= & 1-(1 / N) \int_{0}^{\pi / 2} I(\phi)\left[\sin ^{2} \phi\left[(105 / 16) \cos ^{2} \phi+15 / 24\right]\right. \\
& +\sin \phi \ln \{(1+\sin \phi) / \cos \phi\} \\
& \left.\left((105 / 16) \cos ^{4} \phi-(15 / 4) \cos ^{2} \phi\right)\right] \mathrm{d} \phi
\end{aligned}
$$

where $N=\int_{0}^{\pi / 2} I(\phi) \mathrm{d} \phi$.

The Leadbetter expression for $l(\phi)$ in terms of a series can be written as

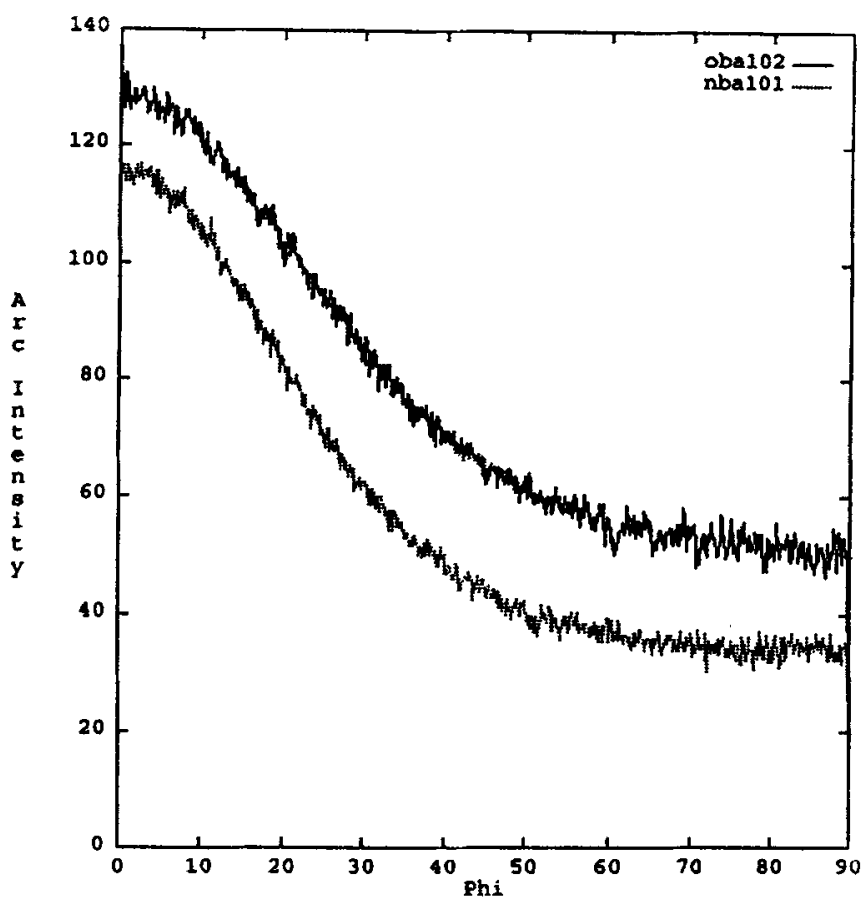

Figure 1. $X$-ray recordings for OBA and NBA at 101 and $102^{\circ} \mathrm{C}$, respectively.

$$
\begin{aligned}
I(\phi) & =f_{0}+2 / 3 f_{2} \cos ^{2} \phi+8 / 15 f_{4} \cos ^{4} \phi \\
& +14 / 35 f_{6} \cos ^{6} \phi+128 / 315 f_{8} \cos ^{8} \phi \\
& +256 / 693 f_{10} \cos ^{10} \phi+\ldots
\end{aligned}
$$

Within the framework of Maier-Saupe model, Levelut group have come up with a novel method wherein there is only one independent parameter and the expression for $l(\phi)$ is given by

$$
\begin{aligned}
I(\phi)= & 1 / Z\left[1+2 / 3 m \cos ^{2} \phi+4 / 15 m^{2} \cos ^{2} \phi\right. \\
& \left.+8 / 105 m^{3} \cos ^{6} \phi+16 / 945 m^{4} \cos ^{8} \phi+\ldots\right],
\end{aligned}
$$

where $Z=4 \pi \int_{0}^{1} \exp \left(m x^{2}\right) \mathrm{d} x$ is the normalization constant. The constants $f$ s in Leadbetter approach and the constant ' $m$ ' in Levelut approach are related to the order parameter via the orientational distribution function via the relations
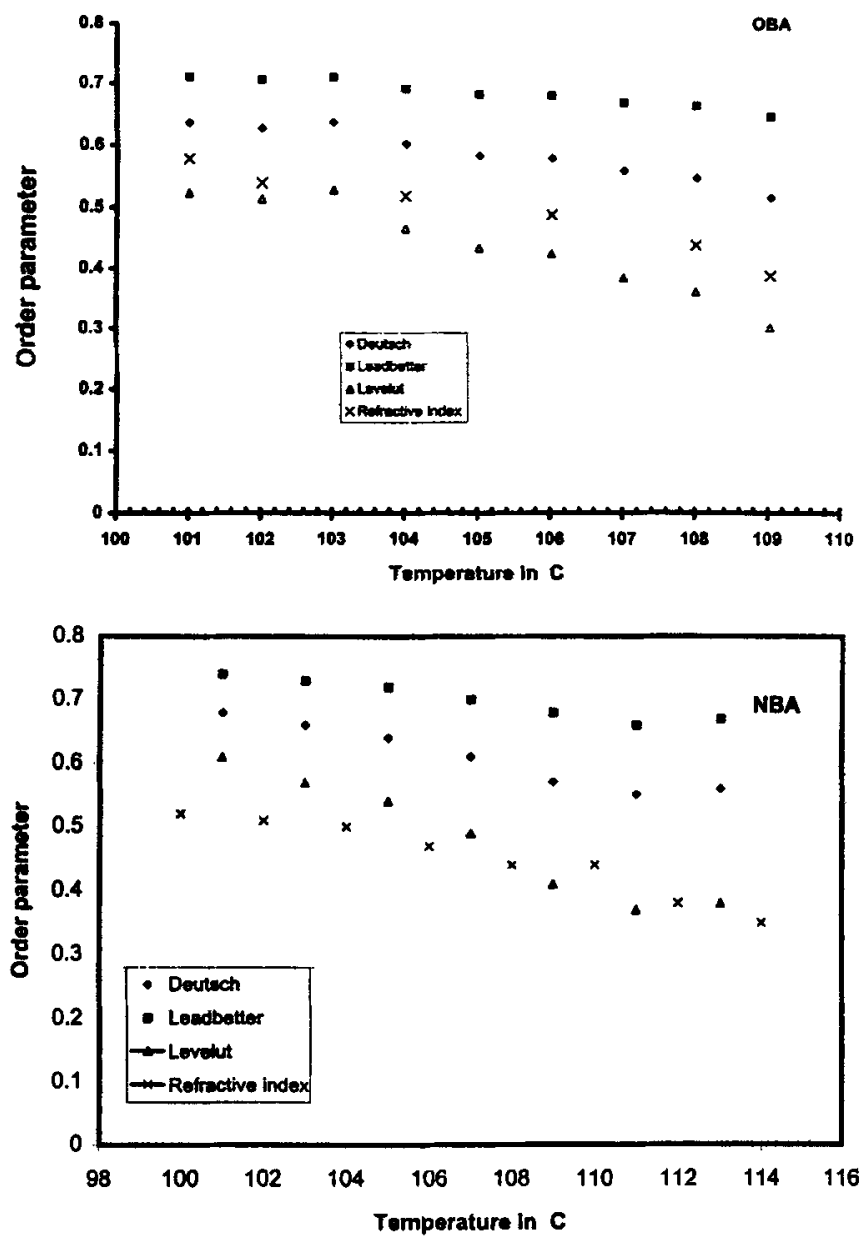

Figure 2. Variation of orientational order parameter $\left\langle P_{2}\right\rangle$ with temperature for OBA and NBA. 
$\left\langle\cos ^{2} \beta\right\rangle=J_{2}(m) / J_{0}(m)$

and

$$
S=\left(3\left\langle\cos ^{2} \beta\right\rangle-1\right) / 2 \text {. }
$$

The relation involving ' $f$ ' is given by

$$
\left\langle\cos ^{2} \beta\right\rangle=\Sigma f_{2 i} /(2 i+3) / \Sigma f_{2 i} / 2 i+1 .
$$

We have written suitable Fortran programmes for the different methods described above to compute the order parameter $\left\langle P_{2}\right\rangle$ using the intensity corresponding to various arc angles. The higher order parameter $\left\langle P_{4}\right\rangle$ has also been computed. The computed values of $\left\langle P_{2}\right\rangle$ at different temperatures are shown in figure 2 along with the order parameter obtained from refractive index data.

\section{Results and discussion}

It is evident from figure 2 that the trend in the variation of $\left\langle P_{2}\right\rangle$ computed from the different methods is the same. However, the values computed by the Deutsch method lies in between the values computed by the Leadbetter et al and Levelut methods. This is due to the truncation of the series to a finite number of terms whereas the solution is exact in Deutsch method. The values obtained from the refractive index data are lower compared to the results from $\mathrm{X}$-ray method. This difference between the X-ray and optical methods arise primarily due to averaging procedure of the orientational distribution function. In X-ray method, it is the local averaging, whereas, it is the local and macroscopic averaging in the optical method. Hence the difference is basically due to the distinction between the microscopic and macroscopic orientational order as observed by Davidson et al (1995), and de Vries (1972). Deutsch method is more reliable because of the fact that it involves computation of orientational order parameter using analytically obtained solutions. Hence under these circumstances, we have used the Deutsch method (3) to estimate the higher order

\begin{tabular}{|c|c|c|c|c|}
\hline \multirow[b]{2}{*}{$T_{c}-T$} & \multicolumn{2}{|c|}{ OBA } & \multicolumn{2}{|c|}{ NBA } \\
\hline & Deutsch & $\begin{array}{l}\text { Refractive } \\
\text { index }\end{array}$ & Deutsch & $\begin{array}{l}\text { Refractive } \\
\text { index }\end{array}$ \\
\hline 12 & - & 0.161 & 0.278 & - \\
\hline 11 & 0.220 & - & 0.233 & 0.098 \\
\hline 10 & 0.210 & 0.127 & 0.259 & - \\
\hline 8 & 0.182 & 0.112 & 0.227 & - \\
\hline 7 & 0.163 & - & 0.117 & 0.071 \\
\hline 6 & 0.160 & 0.092 & 0.134 & - \\
\hline 5 & 0.141 & - & 0.114 & 0.064 \\
\hline 4 & 0.131 & 0.064 & 0.104 & - \\
\hline 3 & 0.106 & - & 0.092 & 0.039 \\
\hline
\end{tabular}

Table 1. Higher order parameter $\left\langle P_{4}\right\rangle$ for OBA and NBA. parameter $\left\langle P_{4}\right\rangle$ and compared with the values of $\left\langle P_{4}\right\rangle$ from the refractive index data (table 1). The necessary distribution functions for $\mathrm{OBA}$ and NBA are given in figure 3. It is observed from table 1 that there is broad agreement between the two methods indicating that the skewness of the distribution function is independent of the type of averaging (local or macroscopic) whereas the variance (width) of the $f(\beta)$ depends on the averaging procedures (de Gennes 1974).
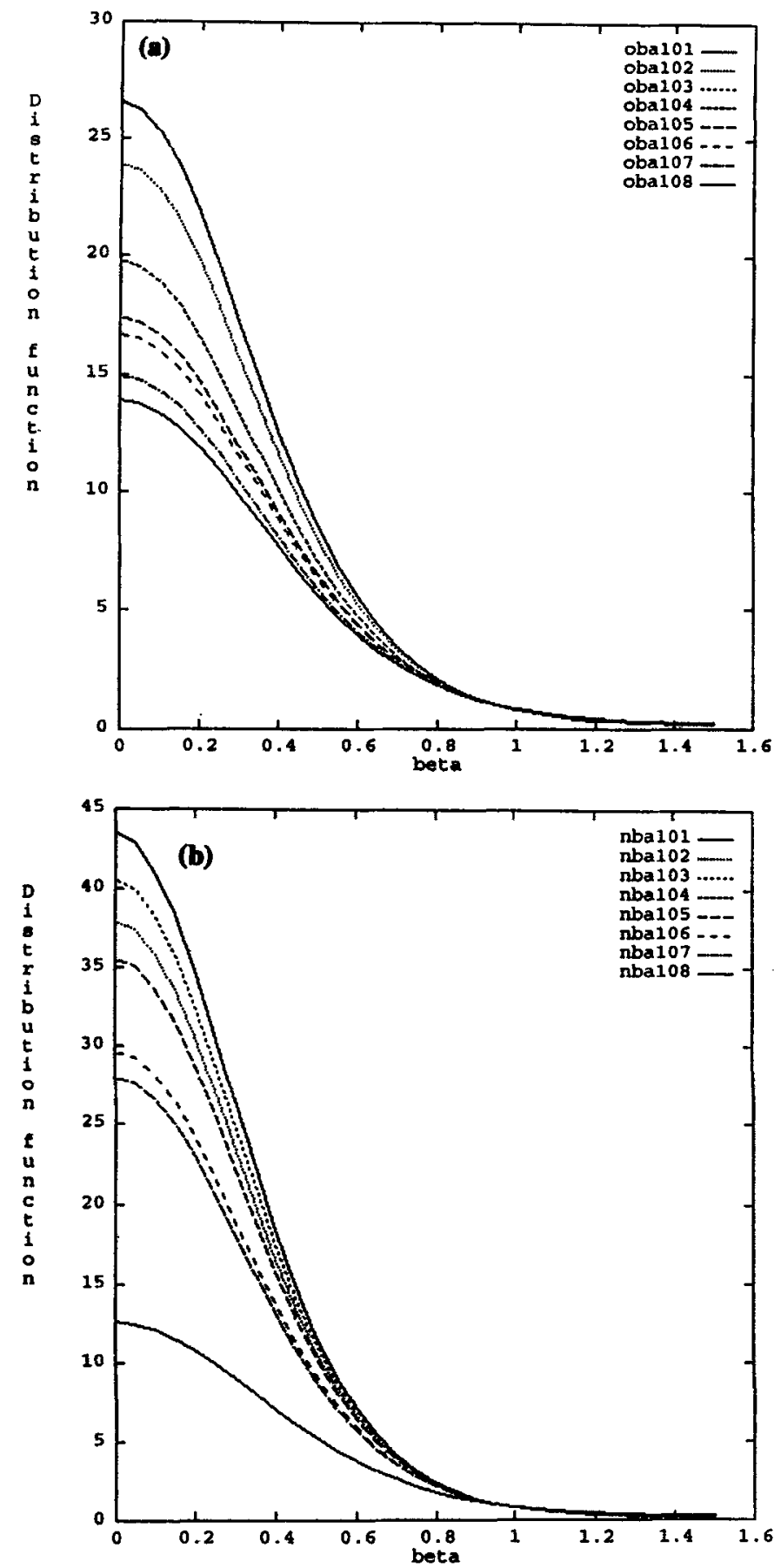

Figure 3. Distribution functions obtained from Deutsch method at various temperatures for (a) OBA and (b) NBA. 


\section{Conclusion}

The orientational order parameters have been estimated from X-ray method using various approaches and compared with the values obtained from the refractive index method for OBA and NBA. The observed differences in orientational parameters have been explained in terms of averaging procedures of $f(\beta)$. It has been pointed out here that the skewness of the $f(\beta)$ is independent of the type of averaging.

\section{Acknowledgements}

Authors thank CSIR, New Delhi for the Project. They also thank Prof. N V Madhusudana and Dr K A Raghunathan, Raman Research Institute, Bangalore for the collection of the X-ray intensity data using the imaging plate system. Authors thank the referees for suggestions to improve the presentation of the paper.

\section{References}

Baumann D, Fan Z X and Haase W 1989 Liq. Cryst. 6239 Bhattacharjee B, Paul S and Paul R 1981 Mol. Phys. 441391
Davidson P, Petermann D and Levelut A M 1995 J. Phys. II France 5113

Delord P and Falgueirettes J 1965 C. R. Acad. Sci. Paris 260 2468

de Gennes P G 1974 The physics of liquid crystals (UK: Oxford Univ. Press)

Deutsch M 1991 Phys. Rev. A44 8264

de Vries A 1972 J. Chem. Phys. 564489

Divya A P, Narayanamurthy K, Madhava M S, Revannasiddaiah D and Somashekar R 1997 Mol. Cryst. Liq. Cryst. 3049

Falgueirettes J 1959 Bull. Soc. Franc. Miner. Crist. 82151

Fan Z X, Buchner S, Haase W and Zachmann H G $1990 \mathrm{~J}$. Chem. Phys. 925099

Haase W, Fan Z X and Muller H J 1988 J. Chem. Phys. 89 3317

Kelkar V K and Paranjape A S 1987 Mol. Cryst. Liq. Cryst. 4139

Leadbetter A J 1979 The molecular physics of liquid crystals (eds) G R Luckhurst and G W Gray (New York: Academic Press)

Leadbetter A J and Norris E K 1979 Mol. Phys. 38669

Leadbetter A J and Wrighton P G 1979 J. Phys. Colloq. France 40 C3-324 